\title{
ENGINEERING SURFACE MICRO-STRUCTURE TO CONTROL FOULING AND HYSTERESIS IN DROPLET BASED MICROFLUIDIC BIOANALYTICAL SYSTEMS
}

\author{
Ashutosh Shastry, Sidhartha Goyal, Aziel Epilepsia, Marianne J. Case, Shaghayegh Abbasi, Buddy Ratner ${ }^{ \pm}$and \\ Karl F. Böhringer \\ Department of Electrical Engineering, ${ }^{ \pm}$Department of Bioengineering, University of Washington \\ Seattle, WA USA
}

\begin{abstract}
This paper presents the principle, fabricated structure, characterization and experimental results obtained for a new class of surfaces-"hydrophobic non-fouling surfaces"-for dropletbased microfluidics. Building on the theory of wetting of rough surfaces, we have developed novel surfaces which are chemically hydrophilic, i.e., the droplet is in contact with a non-fouling hydrophilic material but has high contact angle as a result of thermodynamically stabilized air traps beneath the droplet. This paper also presents the experimental characterization of rough super-hydrophobic surfaces, dynamic measurements of sliding angles of water droplets, and a modeling approach to estimate bounds on contact angle hysteresis - a major dissipative mechanism in droplet based microfluidic systems. A comprehensive study of the dependence of hysteresis on texture parameters is presented to evaluate the current model, propose a modification, and show that the two models-original and modified - provide useful bounds on the hysteresis of the surface.
\end{abstract}

\section{INTRODUCTION}

Microscale bioanalysis offers assay possibilities useful for diagnostic applications and as research tools for biologists. The promise to enable spatially and temporally resolved chemistries [1] has fuelled the emergence of droplet-based "digital" microfluidics. Electrowetting, the electrical approach to droplet manipulation, involves voltage-controlled actuation of droplets on a "hydrophobic" surface. Proteins are ubiquitous components of bioassays. Past studies have confirmed, however, that hydrophobic interactions are involved in protein adsorption [2, 3]. Also, most non-fouling materials are hydrophilic [3]. The use of hydrophobic surfaces therefore presents a challenge. It is important to devise a strategy to minimize protein adsorption on the surface to not only guard against the system being rendered defunct but also to minimize protein loss from the limited quantity present in a droplet.

While minimizing bio-fouling is one worthwhile pursuit, developing low energy schemes of moving droplets is another. Low energy schemes are particularly interesting as they can enable microfluidic platforms with integrated electronics. While drag and contact angle hysteresis are the two energy dissipative mechanisms at work, it is the latter that decides the actuation voltages [4]. Bringing actuation voltages down to the sub-CMOS regime is critical to the development of integrated microfluidic platforms. One route to lower actuation voltages and to reduce energy dissipation involves employing low hysteresis, low drag rough hydrophobic surfaces $[\mathbf{5}, \mathbf{6}]$. Understanding the quantitative relationship between the impeding force of contact angle hysteresis and surface parameters is an important milestone in this pursuit.

We first review the theory of wetting of rough surfaces and then explain the principle of our hydrophobic non-fouling surfaces. Next we detail the fabrication of rough hydrophobic surfaces and the non-fouling hydrophobic surfaces. Experimental work, results and discussion follow. We conclude by summarizing the on-going efforts to complete these studies.

\section{THEORY AND PRINCIPLE}

Consider a rough surface realized by creating pillars of controlled geometry on a smooth surface. Here the roughness is characterized by $r$, the ratio of rough to planar surface area, as explained in Fig. 1 . The basic effect of surface roughness on wetting is modeled by Wenzel's relation (Eq. 1), which relates the apparent contact angle $\theta_{W}$ of a droplet on a rough surface with $r \geq 1$ to Young's intrinsic contact angle $\theta_{i}[7]$.

$$
\cos \theta_{W}=r \cos \theta_{i}
$$

In the Wenzel state the droplet is conformal with the topography, as seen in Fig. 2(b). The droplet can also sit on the pillar tops with air pockets trapped beneath it, as shown in Fig. 2(a). This configuration is referred to as the "Fakir" state. In the Fakir state, the base of the droplet essentially contacts a composite surface of pillar tops and air. The apparent contact angle $\theta_{C B}$ on a composite surface is determined using the Cassie-Baxter relation given in Eq. 2.

$$
\cos \theta_{C B}=\sum_{j} \phi_{j} \cos \theta_{i}
$$

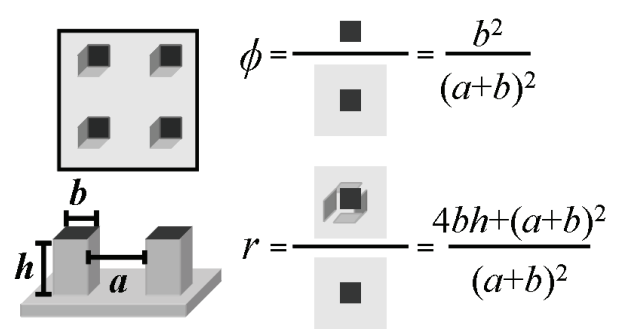

Figure 1: The texture parameters $\phi$ and $r$ are expressed in terms of the design parameters a (gap length), $b$ (pillar size) and $h$ (pillar height), where $\phi$ is the fraction of pillar top area over total horizontal surface area and $r$ is the fraction of total surface area over total horizontal surface area.

Here, $\phi_{j}$ is the surface area fraction and $\theta_{j}$ is the intrinsic contact angle of water with material $j$. For a sessile Fakir droplet, a surface area fraction $\phi$ (Fig. 1) of its base rests on pillar tops that have an intrinsic contact angle of $\theta_{i}$; the remaining surface area fraction of $(1-\phi)$ suspends freely, contacting air with a contact angle of $180^{\circ}$. Substituting these values, the apparent contact angle in the Fakir state-a special case of Cassie-Baxter contact-is readily computed $[7,8]$ to yield Eq. 3 .

$$
\cos \theta_{F}=\phi\left(\cos \theta_{i}+1\right)-1
$$




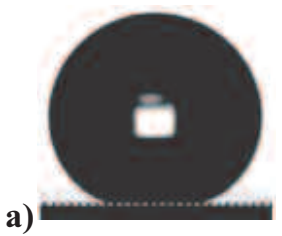

b)

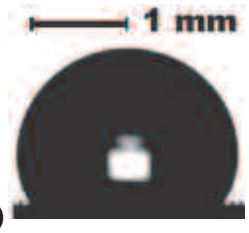

Figure 2: Droplets of volume $5 \mu \mathrm{l}$, on a Teflon-coated silicon surface with $\phi=0.05$ and $r=1.4$ in a) Fakir state with a footprint diameter of $1 \mathrm{~mm}, \theta_{F}=156.6^{\circ}$ (expected: $164.5^{\circ}$ ) and b) Wenzel state with a footprint diameter of $1.96 \mathrm{~mm}, \theta_{W}=118^{\circ}$ (expected: $\left.112.8^{\circ}\right)$. Air pockets are visible between pillars under the Fakir state droplet. Pinning of the droplet edge causes significant deviations from the predicted equilibrium value.

The angles $\theta_{F}$ from Eq. 3 and $\theta_{W}$ from Eq. 1 are equilibrium values of the apparent contact angle in the two states. Equilibrium angles of the droplet are expected when there is no impending movement. When there is impending movement, the difference between the cosines of maximum advancing and minimum receding angles that a droplet makes with a surface is defined as contact angle hysteresis. Hysteresis results from the pinning of the three-phase contact line to the solid surface and is attributed to physical and chemical inhomogeneities [6]. At equilibrium, we notice by comparing $\theta_{F}$ from Eq. 3 and $\theta_{W}$ from Eq. 1 that the Fakir state has a lower energy relative to the Wenzel state (i.e., $\cos \theta_{W}<\cos \theta_{F}$ ) if the following inequality proposed by Bico et al. [8] (Eq. 4) holds true.

$$
\cos \theta_{i}<\frac{\phi-1}{r-\phi}
$$

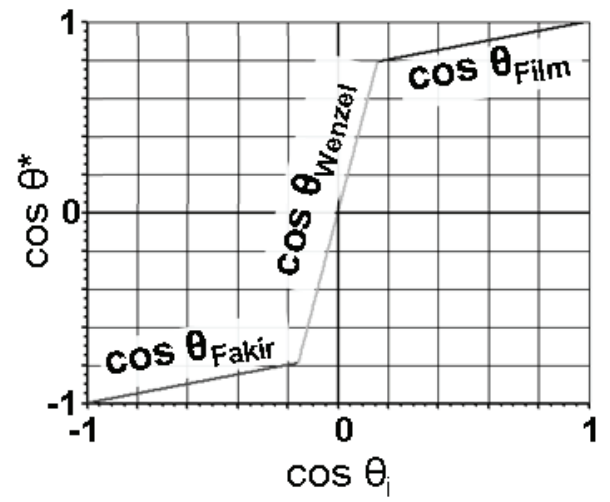

Figure 3: Fakir, Wenzel and Film regimes are shown for a typical rough surface with $r=5$ and $\phi=.25$.

A droplet at a given location on a surface does not spontaneously transit from one state to the other because of the presence of an energy barrier. This energy barrier is analogous to the activation energy of a chemical reaction that prevents spontaneous conversion to products (Fig. 4). This energy barrier is easily estimated [9]. In situations where Eq. 4 does not hold, the energy barrier gives a useful bound on the energy that needs to be coupled to a "metastable" Fakir droplet before risking its transition to the Wenzel state.

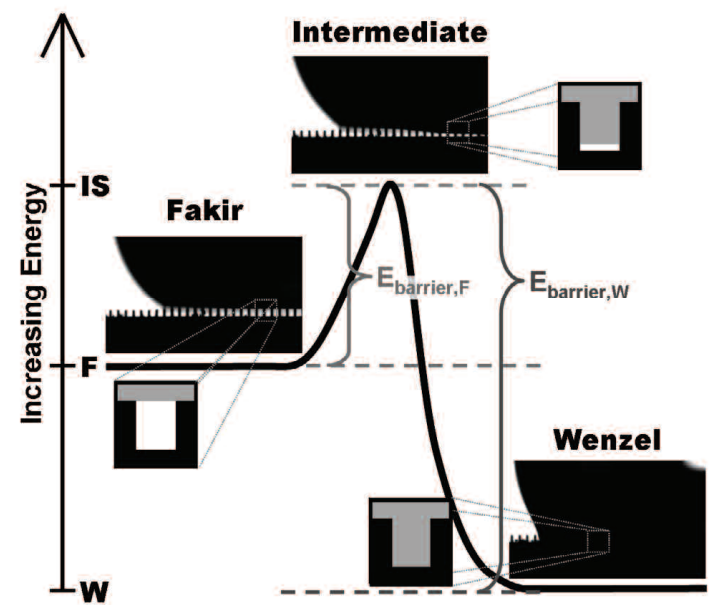

Figure 4: A schematic showing energy levels of the Fakir and Wenzel states. The choice of texture parameters makes the Fakir state metastable in this case. The energy of the intermediate state is calculated by assuming nearly complete penetration of the droplet; only a thin film of air separates the liquid-air and solidair interfaces at the bottom of the valleys. A Fakir droplet needs to overcome the energy barrier $E_{\mathrm{barrier}, \mathrm{F}}=E_{I S}-E_{F}$ to transit to the Wenzel state.

From this analysis, we realize that the contact angle depends only on $\phi$ and $\theta_{i}$-it is independent of the coating on the sidewall. But the energy barrier depends only on the coating of the sidewalls - it is independent of the $\theta_{i}$ of the pillar tops. A Fakir droplet can exist with hydrophilic, non-fouling pillar tops pillar tops but hydrophobic side walls.

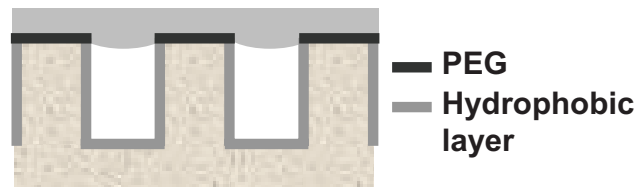

Figure 5: Schematic diagram of liquid deposited on physically and chemically patterned surface. The top surface is hydrophilic nonfouling polyethylene glycol (PEG). The trough and side-walls are hydrophobic. Although the liquid-vapor surface may be curved, the large size of the droplet relative to the spacing between pillars allows the profile to be approximated as spanning straight across surfaces in the derivation.

We therefore proposed composite micro-textured surfaces with hydrophobic troughs and side walls of the pillars and a hydrophilic non-fouling material polyethylene glycol (PEG) on the pillar tops (Fig. 5).

\section{FABRICATION AND EXPERIMENT}

The fabrication process employed for the hydrophobic-non fouling surfaces is detailed in Fig. 6. A thiol-ending molecule formed a self assembled monolayer to provide the hydrophobic coating on gold. Contact angles of the droplets were measured on these surfaces to test the hypothesis. 

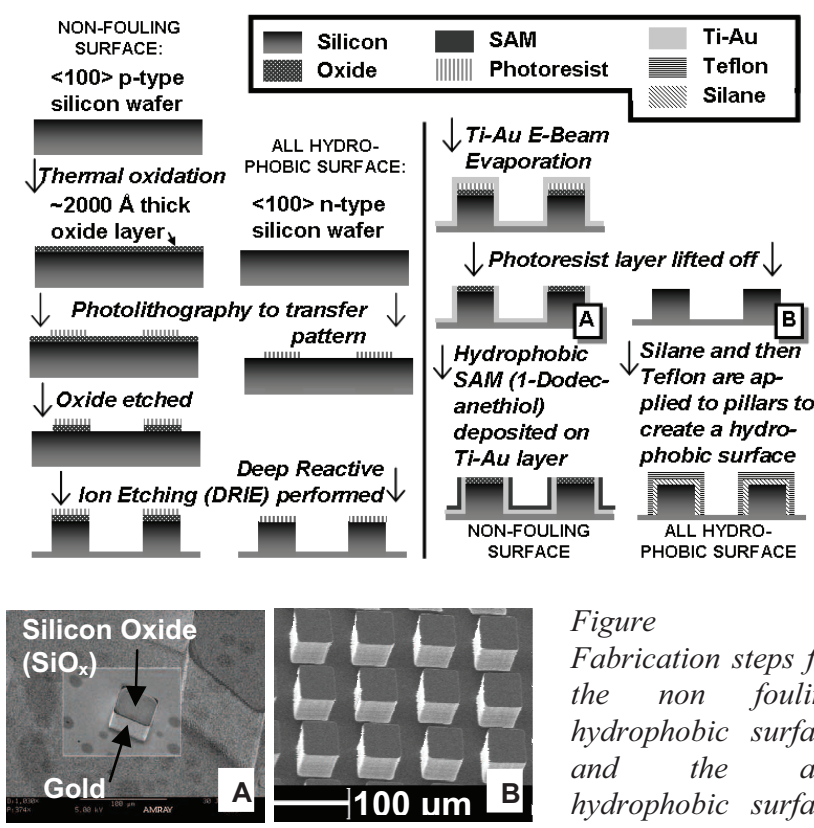

Figure 4: Fabrication steps for the non fouling hydrophobic surface and the allhydrophobic surface (SEM micrograph B), are shown. In SEM micrograph A, the non-conducting oxide shows up dark while conducting metal film (Ti-Au) on the sidewalls of the pillars and on the trough shows up bright.
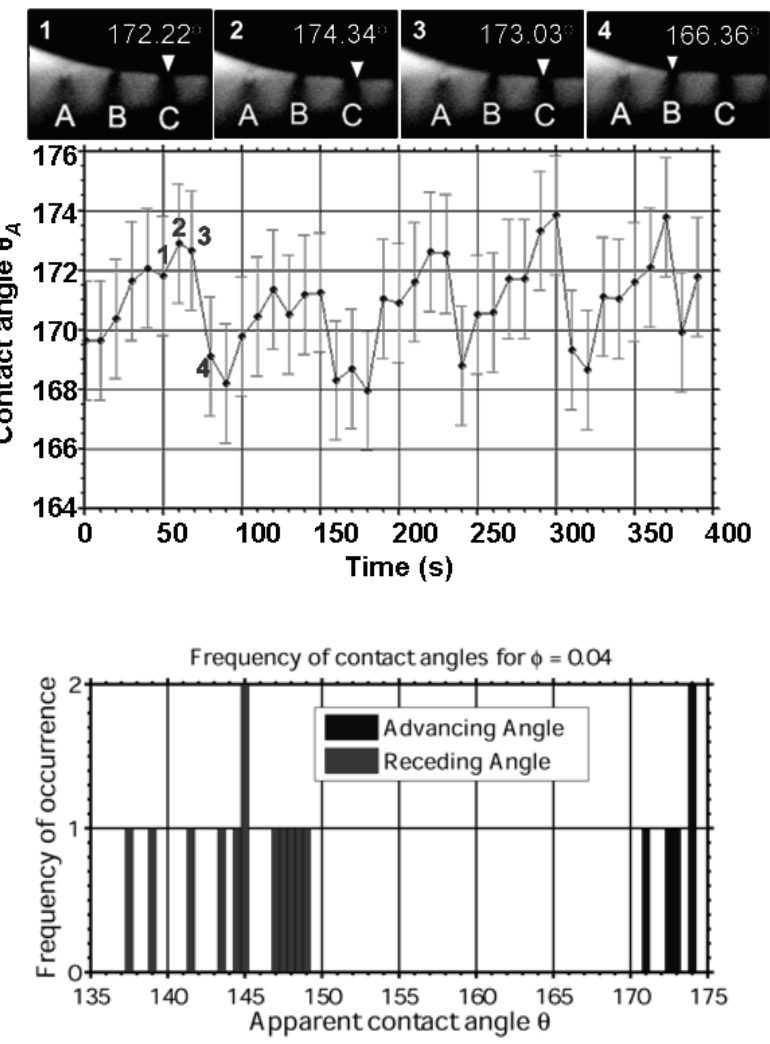

Figure 6: a) The advancing contact angle is plotted as a function of time for a sessile droplet (on a surface with $\phi=0.04$ ) b) Contact angle values in the entire video for the advancing angle measurements were used to create the histogram shown.
We built another set of test surfaces with similar physical texture but uniformly covered with a hydrophobic coating of Teflon AF 1600 as detailed in Fig. 5. These test surfaces were used for hysteresis characterization. Sessile droplets on these surfaces were expanded and contracted by adding and removing water using the syringe pump while a video was recorded at $60 \mathrm{fps}$; contact angles were measured on each frame. As seen in Fig. 6a, the contact angle increases as the advancing edge expands forward but drops precipitously as it eventually lands on the next pillar. For the contracting droplet, the receding angle jumps to a high value as the receding edge snaps off a pillar. Each landing (or snapping) is an experiment and the mode value is reported (Fig. 6b) as the advancing or the receding angle for a surface. Movies were made for surfaces of varying texture ( $\phi$ ranging from 0.025 to 1 ), data plotted, and trends analyzed.

\section{RESULTS AND DISCUSSION}

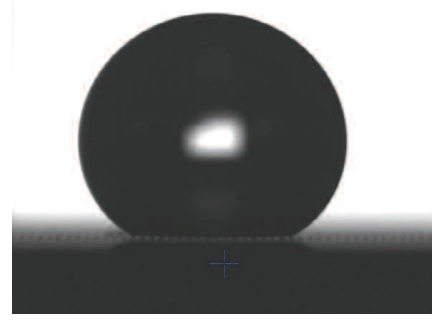

Figure 7: A droplet of volume $7.68 \mu \mathrm{l}$ in Fakir regime with $\theta_{F}=$ $137^{\circ}$. Light below the droplet confirms that it is the Fakir regime.

For the droplet placed on the hydrophobic surface with hydrophilic pillar tops, its contact angle was measured using a goniometer. The light filtering through the air traps beneath the droplet established that it rested as expected, in the Fakir state, contacting only the hydrophilic oxide layer on the pillar tops. It therefore made a high contact angle as seen in Fig. 7. The measurements were repeated over several test surfaces - with a range of pillar widths and gaps-as summed up in Fig. 8.

For the hysteresis experiments, the cosines of advancing and receding angles were observed to decrease linearly with $\phi$-for both the Teflon AF 1600 and FOTS coated surfaces - as evident from Fig. 9. The advancing angle model is $\cos \theta_{A}=-1+\phi\left(1+\cos \theta_{i, A}\right)$, a heuristic relation proposed by $\mathrm{He}$ et al. [11] —obtained by replacing the intrinsic equilibrium contact angle $\theta_{i}$ with the intrinsic advancing angle $\theta_{i, A}$.

These results provide the first experimental validation of this relation. The current model for the receding angle in the Fakir state, $\cos \theta_{R}=2 \phi-1$, is obtained assuming a trailing film remains on the pillar tops [11]. As seen in Fig. 8a, this assumption, originally proposed for hydrophilic surfaces [10], overestimates hysteresis - providing an upper bound (for $\phi>0.1$ ). We re-derived the relation from first principles, heuristically replacing " 1 " $(=\cos 0)$ by $\cos \theta_{I}$ in the coefficient of $\phi$ to obtain $\cos \theta_{R}=\phi\left(1+\cos \theta_{i, R}\right)-1$. This model accounts for partial coverage of pillars by the trailing film - as expected for a hydrophobic coating. Our model provides the lower bound to hysteresis (Fig. 9c). 


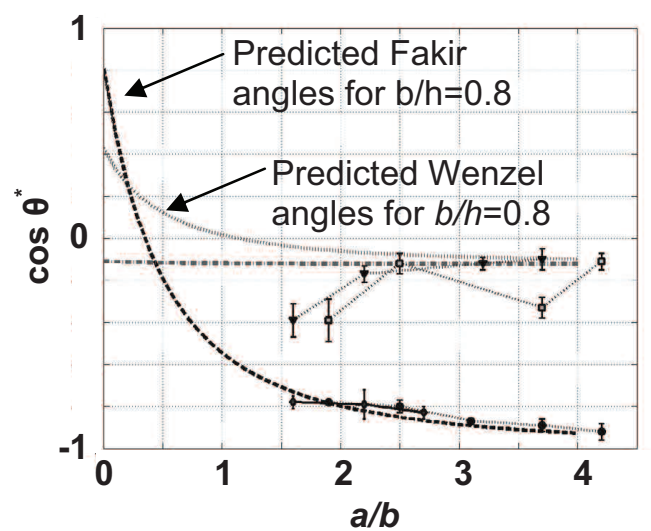

Figure 8: Measured contact angle values for Wenzel and Fakir are plotted along with the predicted angles based on the measured pillar dimension $b$ and spacing $a$. Predicted values for Fakir, Wenzel with $h / b=0.8$, and Wenzel with $h / b=1.9$.
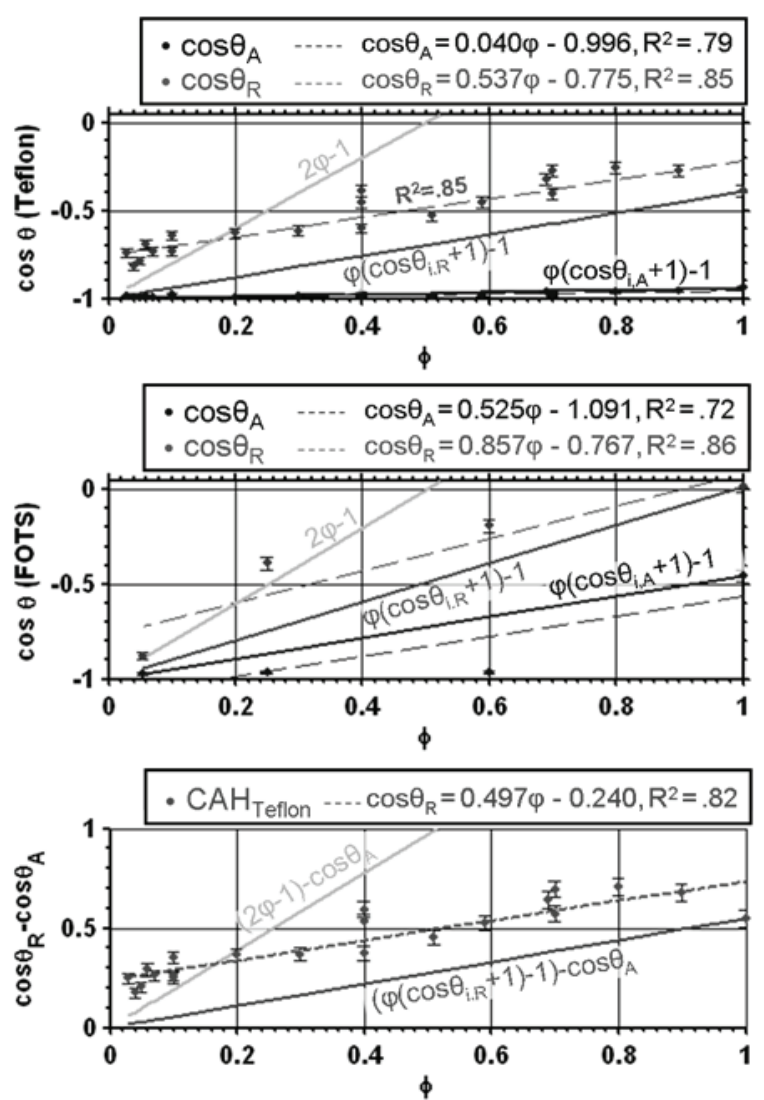

Figure 9: Mode values of advancing and receding contact angles obtained on a) Teflon coated surfaces and b) FOTS coated surfaces are plotted as a function of $\phi$ along with the linear fit lines and model predictions. c) The contact angle hysteresis (CAH) is plotted as a function of $\phi$ for Teflon, as well as model lines and a fit line.

\section{CONCLUSIONS}

We have introduced a new class of non-fouling surfaces and have obtained the "proof-of-concept" results demonstrating hydrophobic surfaces where droplets contact only the in the

"hydrophilic" portions of rough surfaces. The ongoing effort entails quantifying their fouling behavior using radio-labeled protein adsorption on a well characterized PEG layer deposited on the oxide.

For very low $\phi \quad(\phi<0.1)$, study is under way to evaluate our hypothesis that the perimeter per unit area, and not $\phi$, controls the hysteresis behavior. The non-fouling hydrophobic surfaces and bounds on contact angle hysteresis thus mark two important steps towards droplet based open microfluidic systems for bioanalytical applications.

\section{ACKNOWLEDGEMENTS}

This work is funded by NIH Center of Excellence in Genomic Science and Technology grant 1-P50-HG002360-01.

\section{REFERENCES}

[1] A. A. Darhuber and S. M. Troian, "Microfluidic actuation by modulation of surface stresses," Ann. Rev. Fluid. Mech., vol. 37, pp. 425-455, January 2005.

[2] V. Tangpasuthodal, N. Pongchaisirikul, and V. P. Hoven, "Surface modification of chitosan films. Effects of hydrophobicity on protein adsorption," Reviews of Modern Physics, vol. 338, pp. 937-942, 2003.

[3] D. G. Caster and B. D. Ratner, "Biomedical surface science: Foundation to frontiers," Surf. Sci., vol. 500, pp. 28-60, 2002.

[4] F. Mugele and J.-C. Baret, "Electrowetting: from basics to applications," Journal of Physics: Condensed Matter, vol. 17, pp. R705-R774, 2005.

[5] C. Cottin-Bizonne, J.-L. Barrat, L. Bocquet, and E. Charlaix, "Low-friction flows of liquid at nanopatterned interfaces," Nature Materials, vol. 2, p. 237240, April 2003.

[6] D. Quéré, A. Lafuma, and J. Bico, "Slippy and sticky microtextured solids," Nanotechnology, vol. 1, pp. 14-15, 2003.

[7] J. Bico, C. Marzolin, and D. Quéré, "Pearl Drops", Europhysics Letters, vol. 47, pp. 220-226, 1999.

[8] J. Bico, U. Thiele, and D. Quéré, "Wetting of textured surfaces", Colloids and Surfaces A: Physiochemical and Engineering Aspects, vol. 206, pp. 41-46, 2002.

[9] N.A. Patankar, "Transition between Superhydrophobic States on Rough Surfaces", Langmuir, vol.20, pp.7097-7102, 2004.

[10] P. Roura and J. Fort, "Equilibrium of drops on inclined hydrophilic surfaces," Physical Review E, vol.64, no.011601, pp. $1-5,2001$.

[11] B. He, J. Lee, and N. A. Patankar, "Contact angle hysteresis on rough hydrophobic surfaces," Colloid and Surfaces A: Physicochem. Eng. Aspects, vol. 248, pp. 101-104, 2004. 